\title{
Numerical Solution of Fingero-Imbibition Phenomena of Time- Fractional Type in Double Phase Flow through Porous Media
}

\author{
M.S.Prajapati ${ }^{1}$, P.H.Bhathawala ${ }^{2}$ \\ ${ }^{1}$ Associate Professor, Muni. Arts \& U.B.Science College, Mehsana-384002, Gujarat, India \\ ${ }^{2}$ Professor of IT, AURO University, Surat-394510, Gujarat, India
}

\begin{abstract}
We consider the non-linear partial differential equation of time-fractional type describing the spontaneous imbibition of water by an oil-saturated rock (double phase flow through porous media). The fact that oil and water form two immiscible liquid phases and water represents preferentially wet-ting phase are the basic assumption of this work. The Elzaki Transform Decomposition Method is used to obtain the saturation of injected water. We obtain the graphical representation of solution using MATLAB R2007b and Microsoft Excel 2010 with different fractional order $(\alpha>0)$. The aim of this work is to extend the application of Elzaki transform decomposition method suggested by M. Khalid et al. to resolve nonlinear partial differential equations. We apply the proposed method to obtain approximate analytical solutions of the proposed problems. Comparison between the numerical and the exact solutions revealed that (ETDM) is an alternative analytical method for solving nonlinear partial differential equations.
\end{abstract}

Key Words and Phrases: Fractional derivative, Fingero-imbibition, Double phase flow in porous media, immiscible fluid, Elzaki Transform Decomposition Method

\section{Introduction}

In Caputo [6] and He [9], the approach used to account for the effects of changing flux is to embody the effects of memory which has to do with posing problem in terms of fractional calculus. Levy-flight type of transport is a well known diffusion process which is described by a fractional system. Motivated by this idea, we propose a fractional type Fingero-Imbibition phenomena equation in double phase flow through porous media and obtain analytical ap-proximate solution using Elzaki Transform Decomposition Method.

We consider equation of form

$$
{ }^{C} D_{T}^{\alpha} S(X, T)=\left(\frac{\partial S(X, T)}{\partial X}\right)^{2}+S(X, T) \frac{\partial^{2} S(X, T)}{\partial X^{2}}
$$

where ${ }^{C} D_{T}^{\alpha}$ is the Caputo fractional derivative with appropriate initial condition.

When there is difference in the viscosity of two flowing phases due to wetting difference, then we have fingering phenomena. The importance of this phenomenon has gained attention by various concerned fields such as geophysics, geo-hydrology, reservoir engineering etc, with little or no attention to the fractional type.

Generally, for the past three decades, fractional calculus has been considered with great importance due to its various applications in fluid flow, control theory of dynamical systems, chemical physics, electrical networks, and so on. The quest of getting accurate methods for solving resulted non-linear model involving fractional order is of almost concern of many re-searchers in this field today.

Various methods have been put to use successfully to obtain analytical solutions such as Adomian Decomposition Method (ADM) [2, 15, 16], Variational Iteration Method (VIM) [10, 16], Homotopy Perturbation Method (HPM) [8], and EXP-function Method [19] see also [11, 12, 18]. One of the powerful analytical approach to solving non-linear differential equations is Elzaki Transform Decomposition Method [1, 4]. Recent works have been done using this method (HAM) to obtain analytical solutions of some differential equations given improvement on the method $[3,5,7]$.

This paper considers equation (1) subject to some appropriate initial where

${ }^{C} D_{T}^{\alpha} S(\cdot)=\frac{\partial^{\alpha}(\cdot)}{\partial T^{\alpha}}$ is a Caputo fractional differential operator. We obtain numerical results at the end with different values of $X$ and $t$ given the effect of both parameters on solution of fingero-imbibition phenomena of fractional type in double phase flow through porous media. 


\section{Mathematical Analysis}

Considering a finite cylindrical piece of homogeneous porous matrix which is saturated with native liquid A surrounded completely by an impermeable surface except for an end of the cylinder labeled as the imbibitions face $\mathrm{x}=0$. This end is opened to an adjacent formation of injected liquid $\mathrm{B}$. The phenomenon of fingering will occur simultaneously with imbibitions for a less viscous and preferentially wetting phase of liquid B which describes a one-dimensional finger-imbibition phenomenon for which the injection is started by imbibitions and resulting displacement produce instabilities.

We assume that the validity of Darcy's law for the double phase flow system [17] the seepage velocities of wetting phase $\left(V_{w}\right)$ and non-wetting phase $\left(V_{o}\right)$ as

$$
\begin{gathered}
V_{w}=-\left(\frac{K_{w}}{\mu_{w}}\right) K\left[\frac{\partial P_{w}}{\partial x}\right] \\
V_{o}=-\left(\frac{K_{o}}{\mu_{o}}\right) K\left[\frac{\partial P_{o}}{\partial x}\right]
\end{gathered}
$$

Where $K_{w}$ and $K_{o}$ are relative permeability,$P_{w}$ and $P_{o}$ are pressure and $\mu_{w}$ and $\mu_{o}$ are kinetic viscosities (constant) of wetting phase and non-wetting phase respectively and the permeability of homogeneous medium is $\mathrm{K}$. The coordinate $\mathrm{x}$ is measured along the axis of the cylindrical medium, the origin being located at the imbibitions face $\mathrm{x}=0$. We have that

$$
V_{o}=-V_{w}
$$

For a counter current flow. Hence (1) \& (2) give

Mehta [18], gives the definition of capillary pressure $P_{c}$ as

$$
\frac{K_{w}}{\mu_{w}} K \frac{\partial P_{w}}{\partial x}+\frac{K_{o}}{\mu_{o}} K \frac{\partial P_{o}}{\partial x}=0
$$

That is

$$
P_{c}=P_{o}-P_{w}
$$

Equation (4) \& (6) give

$$
\frac{\partial P_{c}}{\partial x}=\frac{\partial P_{o}}{\partial x}-\frac{\partial P_{w}}{\partial x}
$$

$$
\left(\frac{K_{w}}{\mu_{w}}+\frac{K_{o}}{\mu_{o}}\right) \frac{\partial P_{w}}{\partial x}+\frac{K_{o}}{\mu_{o}} \frac{\partial P_{c}}{\partial x}=0
$$

From here, using (7), we can write (1) \& (2) as

$$
V_{w}=K\left(\frac{K_{w}}{\mu_{w}}\right)\left(\frac{K_{o}}{\mu_{o}}\right) \frac{\partial P_{c}}{\partial x}\left(\frac{K_{w}}{\mu_{w}}+\frac{\partial K_{o}}{\partial \mu_{o}}\right)^{-1}
$$

For wetting phase, equation of continuity is given by

$$
\phi \frac{\partial S_{w}}{\partial t}+\frac{\partial V_{w}}{\partial x}=0
$$

Where $S_{w}$ is the saturation of the wetting phase and $\phi$ is the porosity of the medium. Substituting the value of $V_{w}$ of (8) into (9), we obtain

$$
\phi \frac{\partial S_{w}}{\partial t}+\frac{\partial}{\partial x}\left[K \frac{K_{w} K_{o}}{K_{w} \mu_{o}+K_{o} \mu_{w}} \frac{\partial P_{c}}{\partial x}\right]=0
$$

Equation (10) is a non-linear partial differential equation that describes that the finger-imbibition phenomenon of two immiscible fluids flow through homogeneous porous cylindrical medium with impervious bounding surface on three sides.

We assume standard forms of for the analytical relationship between the relative permeability, phase saturation and capillary pressure phase saturation knowing that fictitious relative permeability is the function of displacing fluid saturation.

Where $\xi=1.11$

$$
K_{o}=1-\xi S_{w}
$$

$$
\begin{gathered}
K_{w}=S_{w} \\
P_{c}=\beta S_{w}
\end{gathered}
$$

The model we are considering involves water and viscous oil so we have

$$
\frac{K_{w} K_{o}}{K_{w} \mu_{o}+K_{o} \mu_{w}} \approx \frac{K_{o}}{\mu_{o}}=\frac{1-\xi S_{w}}{\mu_{o}}=\frac{S}{\mu_{o}}, \text { where } S=1-\xi S_{w}
$$

Hence by substituting (14), (13), (12) and (11) into (10), we arrived at

$$
\frac{\partial S_{w}}{\partial t}=\frac{K \beta}{\mu_{o} \phi} \frac{\partial}{\partial x}\left[\left(1-\xi S_{w}\right) \frac{\partial S_{w}}{\partial x}\right]
$$

For a dimensionless form of (15), we choose the following new variables

$$
X=\frac{x}{L} \text { and } T=\frac{K \beta}{\mu_{o} \phi L^{2}} t
$$


We get

$$
\frac{\partial S_{w}}{\partial T}=\frac{\partial}{\partial X}\left[\left(1-\xi S_{w}\right) \frac{\partial S_{w}}{\partial X}\right] \quad \& S_{w}(X, 0)=f(X)=e^{-X}
$$

The choice of initial condition is due to the fact that the saturation of injected water decreases exponentially when X increases (Mehta [14]).

\section{Elzaki Transform}

The Basic definition of Elzaki Transform is given below

A New integral transform called Elzaki transform ([1], [20]) defined for functions of exponential order is proclaimed. We consider functions in the set A defined by

$$
A=\left\{f(t) / M, K_{1}, K_{2}>0,|f(t)|<M e^{\frac{|t|}{K_{j}}}, \text { if } t \in(-1)^{j} X[0, \infty)\right\}
$$

Definition: If $f(t)$ is function defined for all $t \geq 0$, its Elzaki transform is the integral of $f(t)$ times $e^{-\frac{t}{v}}$ from $t=0$ to $\infty$. It is a function of $v$ and is defined as

$$
\begin{gathered}
E[f(t)]=T(v)=v \int_{0}^{\infty} f(t) e^{-\frac{t}{v}} d t \quad v \in\left(K_{1}, K_{2}\right) \\
\text { or equivalently } T(v)=v^{2} \int_{0}^{\infty} f(v t) e^{-t} d t \quad K_{1}, K_{2}>0
\end{gathered}
$$

Theorem 1: Elzaki transform amplifies the coefficients of the power series function,

$$
f(t)=\sum_{n=0}^{\infty} a_{n} t^{n}
$$

On the new integral transform "Elzaki Transform" is

$$
E\{f(t)]=T(v)=\sum_{n=0}^{\infty} n ! a_{n} v^{n+2}
$$

Theorem:2 Let $f(t)$ be in $\mathrm{A}$ and Let $T_{n}(v)$ denote Elzaki transform of nth derivative, $f^{n}(t)$ of $f(t)$, then for $n \geq 1$,

$$
T_{n}(v)=\frac{T(v)}{v^{n}}-\sum_{k=0}^{n-1} v^{2-n+k} f^{(k)}(0)
$$

To obtain Elzaki transform of partial derivative we use integration by parts, and then we have

$$
\begin{array}{r}
E\left(\frac{\partial f(x, t)}{\partial t}\right)=\frac{1}{v} T(x, v)-v f(x, 0), \\
E\left(\frac{\partial^{2} f(x, t)}{\partial t^{2}}\right)=\frac{1}{v^{2}} T(x, v)-f(x, 0)-v \frac{\partial f(x, 0)}{\partial t}
\end{array}
$$

Properties of Elzaki transform
1. $E(1)=v^{2}$
2. $E\left(t^{n}\right)=n ! v^{n+2}$
3. $E(t)=v^{3}$
4. $E^{-1}\left(v^{n+2}\right)=\frac{t^{n}}{n !}$

\section{Elzaki Transform Decomposition Method for PDEs}

A new modified method for solving partial differential equations. To illustrate the basic idea of this method, we consider a general non-linear non-homogeneous partial differential equation

$$
\frac{\partial^{m} u(x, t)}{\partial t^{m}}+R u(x, t)+N u(x, t)=g(x, t)
$$

Where $\mathrm{m}=1,2,3$, with the initial conditions

$$
\left.\frac{\partial^{m} u(x, t)}{\partial t^{m}}\right|_{t=0}=f_{m-1}(x), \quad m=1,2,3, . .
$$

Where $\frac{\partial^{m} u(x, t)}{\partial t^{m}}$ is the partial derivative of the function $u(x, t)$ of order $m, \mathrm{R}$ is the linear differential operator, $\mathrm{N}$ represents the general nonlinear differential operator, and $\mathrm{g}(\mathrm{x}, \mathrm{t})$ is the source term. Applying the Elzaki Transform (denoted in this paper by E) on both side of Eq. (21), we get

$$
E\left[\frac{\partial^{m} u(x, t)}{\partial t^{m}}\right]+E[R u(x, t)]+E[N u(x, t)]=E[g(x, t)]
$$


Using the properties of Elzaki Transform, we obtain

$v^{-m} E[u(x, t)]=\sum_{k=0}^{m-1} v^{2-m+k} \frac{\partial^{k} u(x, 0)}{\partial t^{k}}+E[g(x, t)]-E[R u(x, t)+N u(x, t)]$,

where $m=1,2,3, \ldots \quad$ And thus, we have

$E[u(x, t)]=\sum_{k=0}^{m-1} v^{2+k} \frac{\partial^{k} u(x, 0)}{\partial t^{k}}+v^{m} E[g(x, t)]-v^{m} E[R u(x, t)+N u(x, t)]$

Operating the inverse transform on both sides of Eq. (25), we get

$$
u(x, t)=G(x, t)-E^{-1}\left(v^{m} E[R u(x, t)+N u(x, t)]\right)
$$

Where $\mathrm{G}(\mathrm{x}, \mathrm{t})$ represents the term arising from the source term and the prescribed initial conditions.

The Second step in Elzaki Transform Decomposition Method, is that we represent the solution as an infinite series given below

$$
u(x, t)=\sum_{n=0}^{\infty} u_{n}(x, t)
$$

And the nonlinear term can be decomposed as

$$
N u(x, t)=\sum_{n=0}^{\infty} A_{n}
$$

Where $A_{n}$ are Adomian polynomials [30] of $u_{0}, u_{1}, u_{2}, \ldots, u_{n}$ and it can be calculated by the formula given below

$$
A_{n}=\frac{1}{n !} \frac{\partial^{n}}{\partial \lambda^{n}}\left[N\left(\sum_{i=0}^{\infty} \lambda^{i} u_{i}\right)\right]_{\lambda=0}, n=0,1,2, \ldots
$$

Substituting (11) and (12) in (10), we have

$$
\sum_{n=0}^{\infty} u_{n}=G(x, t)-E^{-1}\left[u^{m} E\left[R \sum_{n=0}^{\infty} u_{n}+\sum_{n=0}^{\infty} A_{n}\right]\right]
$$

On comparing both side of the equation (30), we get

$$
\begin{gathered}
u_{0}(x, t)=G(x, t) \\
u_{1}(x, t)=-E^{-1}\left[u^{m} E\left[R u_{0}(x, t)+A_{0}\right]\right] \\
u_{2}(x, t)=-E^{-1}\left[u^{m} E\left[R u_{1}(x, t)+A_{1}\right]\right] \\
u_{3}(x, t)=-E^{-1}\left[u^{m} E\left[R u_{2}(x, t)+A_{2}\right]\right]
\end{gathered}
$$

In general, the recursive relation is given as

Where $m=1,2,3$ and $n \geq 0$.

$$
u_{n+1}(x, t)=-E^{-1}\left[u^{m} E\left[R u_{n}(x, t)+A_{n}\right]\right]
$$

Finally, we approximate the analytical solution $u(x, t)$ by truncated series

$$
u(x, t)=\lim _{N \rightarrow \infty} \sum_{n=0}^{N} u_{n}(x, t)
$$

The above series solutions generally converge very rapidly .

Solution of the Equation (31) by ETDM

\section{Solution by Elzaki Transform decomposition Method}

$$
\begin{aligned}
& E\left[\frac{\partial S_{w}}{\partial T}\right]=E\left[\left(1-\xi S_{w}\right)\left(\frac{\partial^{2} S_{w}}{\partial x^{2}}\right)-\xi\left(\frac{\partial S_{w}}{\partial X}\right)^{2}\right] \\
& \frac{E\left[S_{w}(X, T)\right]}{v}-v S_{w 0}(X, 0)=E\left[\left(1-\xi S_{w}\right)\left(\left(S_{w}\right)_{X X}\right)-\xi\left(\left(S_{w}\right)_{X}\left(S_{w}\right)_{X}\right)\right]
\end{aligned}
$$

Here both term are nonlinear

$$
\left[S_{w}(X, T)\right]=v^{2} S_{w 0}(X, 0)+E^{-1}\left[v E\left[\sum_{n=0}^{\infty} A_{n}(S)+\sum_{n=0}^{\infty} B_{n}(S)\right]\right]
$$

From the initial condition (A2) \& equation (18) we get

$$
\begin{aligned}
& S_{w 0}(X, T)=e^{-X} \\
S_{w 1}(X, T) & =E^{-1}\left[v E\left[A_{0}(S)+B_{0}(S)\right]\right] \\
S_{w 2}(X, T) & =E^{-1}\left[v E\left[A_{1}(S)+B_{1}(S)\right]\right] \\
S_{w 3}(X, T) & =E^{-1}\left[v E\left[A_{2}(S)+B_{2}(S)\right]\right]
\end{aligned}
$$


Where

$$
\begin{gathered}
A_{0}(S)=\left(1-\xi S_{w 0}\right)\left(S_{w 0}\right)_{X X} \\
A_{2}(S)=\left(1-\xi S_{w 0}\right)\left(S_{w 2}\right)_{X X}+\left(1-\xi S_{w 1}\right)\left(S_{w 1}\right)_{X X}+\left(1-\xi S_{w 2}\right)\left(S_{w 0}\right)_{X X} \\
\ldots \ldots \\
B_{0}(S)=\xi\left[\left(S_{w 0}\right)_{X}\left(S_{w 0}\right)_{X}\right] \\
B_{1}(S)=\xi\left[\left(S_{w 0}\right)_{X}\left(S_{w 1}\right)_{X}+\left(S_{w 1}\right)_{X}\left(S_{w 0}\right)_{X}\right] \\
B_{2}(S)=\xi\left[\left(S_{w 0}\right)_{X}\left(S_{w 2}\right)_{X}+\left(S_{w 1}\right)_{X}\left(S_{w 1}\right)_{X}+\left(S_{w 2}\right)_{X}\left(S_{w 0}\right)_{X}\right]
\end{gathered}
$$

From above results we get

$$
\begin{gathered}
S_{w 1}(x, t)=\left(e^{-X}-2 \xi e^{-2 X}\right) t \\
S_{w 2}(x, t)=\left(18 \xi^{2} e^{-3 X}-12 \xi e^{-2 X}+2 e^{-X}\right) t^{2} \\
S_{w 3}(x, t)=\left(-304 \xi^{3} e^{-4 X}+282 \xi^{2} e^{-3 X}-66 \xi e^{-2 X}+4 e^{-X}\right) t^{3}
\end{gathered}
$$

\begin{tabular}{|c|c|c|c|c|c|c|c|c|c|c|c|}
\hline $\mathrm{X} / \mathrm{t}$ & 0 & 0.1 & 0.2 & 0.3 & 0.4 & 0.5 & 0.6 & 0.7 & 0.8 & 0.9 & 1 \\
\hline 0 & 1 & 1 & 1 & 1 & 1 & 1 & 1 & 1 & 1 & 1 & 1 \\
\hline 0.1 & 0.8494 & 0.8528 & 0.8237 & 0.7795 & 0.7414 & 0.7151 & 0.6984 & 0.6879 & 0.6812 & 0.6769 & 0.674 \\
\hline 0.2 & 0.7132 & 0.72 & 0.6774 & 0.611 & 0.5531 & 0.5129 & 0.4872 & 0.4709 & 0.4605 & 0.4536 & 0.4491 \\
\hline 0.3 & 0.59 & 0.5995 & 0.5532 & 0.4793 & 0.4142 & 0.3686 & 0.3393 & 0.3205 & 0.3085 & 0.3005 & 0.2951 \\
\hline 0.4 & 0.4784 & 0.4897 & 0.4458 & 0.3739 & 0.3098 & 0.2646 & 0.2354 & 0.2165 & 0.2043 & 0.1962 & 0.1907 \\
\hline 0.5 & 0.3775 & 0.3893 & 0.3513 & 0.2872 & 0.2295 & 0.1885 & 0.1619 & 0.1445 & 0.1333 & 0.1256 & 0.1205 \\
\hline 0.6 & 0.2862 & 0.2974 & 0.2669 & 0.2141 & 0.166 & 0.1316 & 0.1091 & 0.0944 & 0.0848 & 0.0782 & 0.0738 \\
\hline 0.7 & 0.2036 & 0.2132 & 0.1908 & 0.151 & 0.1143 & 0.0879 & 0.0705 & 0.0591 & 0.0516 & 0.0464 & 0.0429 \\
\hline 0.8 & 0.1288 & 0.1359 & 0.1216 & 0.0953 & 0.0709 & 0.0531 & 0.0414 & 0.0336 & 0.0285 & 0.0249 & 0.0225 \\
\hline 0.9 & 0.0612 & 0.065 & 0.0582 & 0.0454 & 0.0333 & 0.0245 & 0.0186 & 0.0147 & 0.0121 & 0.0103 & 0.009 \\
\hline 1 & 0 & 0 & 0 & 0 & 0 & 0 & 0 & 0 & 0 & 0 & 0 \\
\hline
\end{tabular}

we can generated other polynomials by putting different values in equation (34)

$$
\begin{aligned}
& S_{w}(x, t)=S_{w 0}(x, t)+S_{w 1}(x, t)+S_{w 2}(x, t)+\cdots \\
& S_{w}(x, t)=e^{-X} t^{0}+\left(e^{-X}-2 \xi e^{-2 X}\right) t+\left(18 \xi^{2} e^{-3 X}-12 \xi e^{-2 X}+2 e^{-X}\right) t^{2}+ \\
& \left(-304 \xi^{3} e^{-4 X}+282 \xi^{2} e^{-3 X}-66 \xi e^{-2 X}+4 e^{-X}\right) t^{3}+\cdots
\end{aligned}
$$

\section{Table and Figure}

The following table shows the approximate solution for saturation of injected liquid for different values of $\mathrm{x}$ at different time using ETDM

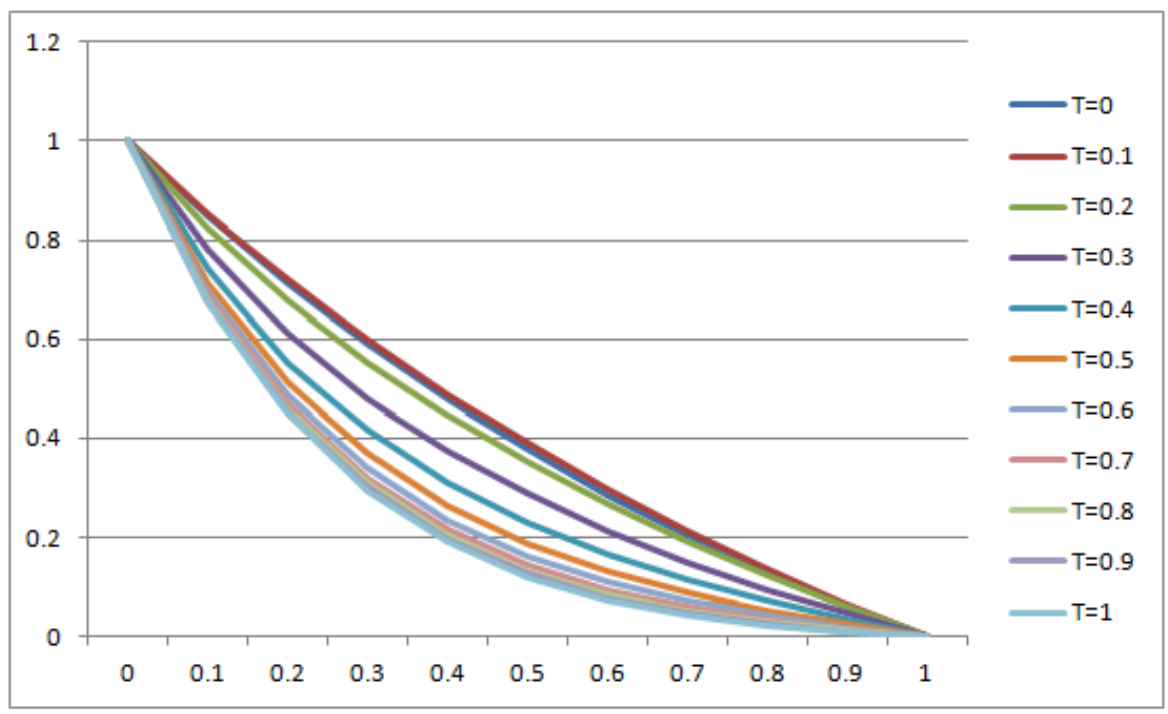




\section{Discussion and conclusion}

The basis for many scientific and engineering applications is now depending largely on the study of physics of flow through porous media. The applications ranges from hydrologist in his study of the migration of underground water, the petroleum engineer in his study of movement of oil, gas and water through the reservoir of oil or gas field or filtration processes. It has applications in ceramic engineering and oil recovery process. In the recent years, the odea of fractional order have been introduced. In the differential equations which have deep meaning and inexhaustible applications. Here, we have studied the fractional Fingero-imbibition equation to model the spontaneous imbibition of water by an oil-saturated rock in a double phase flow through porous media. Elzaki Transform Decomposition Method (ETDM) was implemented to obtain the approximate analytical solution of the problem. The results are shown in Figure. The numerical simulations are obtained using MATLAB R2007b and Microsoft Excel 2010.

\section{References}

[1]. T.M.Elzaki and S.M.Elzaki and E.A.Elnour, On the New Integral Transform “ Elzaki Transform” Fundamental Properties Investigations and Applications, Glo.J.Math. Sci.,4, (2012), 1-13.

[2]. O. Abdulaziz, I. Hashim, M. S. H. Chowdhury, and A. K. Zulkifle. Assessment of decomposition method for linear and nonlinear fractional differential equations, Far East Journal of Applied Mathematics, vol. 28, no. 1, pp. 95-112, 2007.

[3]. O. Abdulaziz, I. Hashim, and A. Saif. Series Solution of Time-Fractional PDEs by Homotopy Analysis Method, Hindawi Publishing Corporation, Differential Equations and Nonlinear Mechanics, pps. 16, 2008. REFERENCES 228

[4]. T.M.Elzaki and E.M.A. Hilal, Homotopy Perturbation and Elzaki Transform for Solving Nonlinear Partial Differential Equations, Math. Theor. Mod., 2, (2012), 33-42.

[5]. A. S. Bataineh, M. S. M. Noorani, and I. Hashim. Approximate solutions of singular twopoint BVPs by modified homotopy analysis method, Physics Letters A, vol. 372, no. 22, pp. 4062-4066, 2008.

[6]. M. Caputo. Diffusion of fluids in porous media with memory, Geothermics 28, 113-130, 1999.

[7]. G. Domairry and M. Fazeli. Homotopy analysis method to determine the fin efficiency of convective straight fins with temperaturedependent thermal conductivity, Communications in Nonlinear Science and Numerical Simulation, vol. 14, no. 2, pp. 489-499, 2009.

[8]. J.-H. He. Application of homotopy perturbation method to nonlinear wave equations, Chaos, Solitons and Fractals, vol. 26, no. 3, pp. 695-700, 2005.

[9]. J.-H. He. Approximate analytical solution for seepage flow with fractional derivatives in porous media, Computer Methods in Applied Mechanics and Engineering. 167, 57-68, 1998.

[10]. J.-H. He and X.-H. Wu. Construction of solitary solution and compacton-like solution by variational iteration method, Chaos, Solitons and Fractals, vol. 29, no. 1, pp. 108-113, 2006.

[11]. C. H. C. Hussin and A. Kilicman. On the Solution of Fractional Order Nonlinear Boundary Value Problems By Using Differential Transformation Method, European Journal of Pure and Applied Mathematics, vol. 4, No. 2, 174-185. 2011.

[12]. O.S. Iyiola. Solving k-Fractional Hilfer Differential Equations via Combined Fractional Integral Transform Methods, British Journal of Mathematics and Computer Science, vol. 4, No. 10, 1427-1436, 2014

[13]. ] S.-J. Liao. An approximate solution technique not depending on small parameters: a special example, International Journal of Non-linear Mechanics, vol. 30, no. 3, pp. 371-380, 1995.

[14]. M.N. Mehta. An asymtotic expansion in fluid flow through porous media; PhD Thesis: S.G. University, Surat (India), 1978.

[15]. R. Meher, M. N. Mehta, and S. K. Meher. Adomian Decomposition Approach to FingeroImbibition Phenomena in Double Phase Flow through Porous Media, International Journal of Applied Mathematics and Mechanics. 6 (9): 34-46, 2012.

[16]. S. Momani and Z. Odibat. Analytical approach to linear fractional partial differential equations arising in fluid mechanics, Physics Letters A, vol. 355, no. 4-5, pp. 271-279, 2006.

[17]. A. E. Scheidegger. The Physics of Flow through Porous Media, The Macmillan Co., New York, 1960

[18]. V. Turut1 and N. Güzel. On solving Partial Differential Equations of Fractional Order by Using the Variational Iteration Method and Multivariate Padé Approximations, European Journal of Pure and Applied Mathematics, Vol. 6, No. 2, 147-171. 2013.

[19]. X.-H. Wu and J.-H. He. EXP-function method and its application to nonlinear equations, Chaos, Solitons and Fractals, vol. 38, no. 3, pp. 903-910, 2008.

[20]. E.M. Abd Elmohmoud and T.M.Elzaki, Elzaki Transform of Derivative Expressed by Heaviside Function, W. Appl. Sci. J., 32, (2014), 1686-1689. 